\title{
Flujo espiratorio máximo en niños asmáticos: Casos y controle.
}

Peak expiratory flow rate in asthmatic children: Case/control study.

\author{
Recabarren Lozada Arturo ${ }^{1}$, Apaza Atencio Nelson²
}

${ }^{1}$ Residente de Neumología Pediátrica. Universidad Peruana Cayetano Heredia. Hospital Nacional Cayetano Heredia.

${ }^{2}$ Profesor Principal, Universidad Nacional de San Agustín, Médico Jefe del Servicio de Pediatría, Hospital Regional Honorio Delgado. Arequipa.

\section{RESUMEN}

Con el fin de determinar las variaciones del Flujo Espiratorio Máximo (PEF), se estudiaron a 38 niños asmáticos (CASOS) en período intercrítico de la enfermedad y a 38 niños sanos (CONTROLES), de ambos sexos comprendidos entre los 5 y 15 años de edad. Los niños asmáticos fueron clasificados por parámetros clínicos en determinado grado de severidad de asma bronquial, determinando el PEF de cada niño objeto de estudio conel mini-Wright Peak Flow Meter, en 2 registros diarios a los 06 y 18 horas, durante 7 días consecutivos, obteniendo la variabilidad del mismo. Se encuentra diferencia en la variabilidad global de niños asmáticos de todos los grados de severidad de la enfermedad comprada con la de los niños normales, con diferencia estadística altamente significativa $(p<0.000001)$. Las variaciones diurnas del PEF ayuda en el diagnóstico del asma bronquial y también son útiles para realizar la catalogación de severidad de la enfermedad. El PEF correlaciona bien con los síntomas presentados por los pacientes y por lo tanto guarda correspondencia con la Hiperreactividad bronquial (HRB) del niño asmático. Postulamos que un niño con historia clínica sugestiva, una variabilidad global mayor del $8 \%$ indica que el diagnóstico de asma es altamente probable (Rev Med Hered 1995; 6: 76-82).

PALABRAS CLAVE: Flujo Espiratorio Máximo (PEF), variabilidad del PEF, asma niños.

\section{SUMMARY}

To determine variations in the Peak Expiratory Flow Rate (PEFR), we studied 38 asthmatic children during intercritical period and 38 healthy children, between 5 and 15 years old. Asthmatic children were classified according to clinic parameters in different levels of severity; using the PEFR measured with the mini-Wright Peak Flow Meter at 6 and 18 hours during 7 consecutive days with its corresponding variability. Difference was found in the global variability of asthmatic children in all severity levels, in contrast with healthy children, with statistic difference $(p<0.000001)$. Diurnal variations on PEFR helps in the diagnosis of asthma and its corresponding degree of severity disease. PEFR correlates good 
with symptoms and airway responsiveness in asthmatic children. We postulate that children with sugestive history and a global variability greater than $8 \%$ have a very probable diagnosis of asthma. (Rev Med Hered 1995; 6: 76-82).

KEY WORDS: Peak expiratory flow rate (PEFR), PEFR variability, asthma, children.

\section{INTRODUCCION}

El asma bronquial continúa siendo una de la enfermedades más comunes a nivel mundial, y existen evidencia que su prevalencia y morbi-mortalidad se encuentra en incremento, a pesar de su mejor reconocimiento y tratamiento (1-3).

Aunque existe diferentes pruebas y determinaciones analíticas de gran utilidad para su diagnóstico, muchas veces adolece de un método auxiliar que sea fácil de realizar, sobre todo cuando se requieren mediciones repetidas (4-6).

Tradicionalmente, el diagnóstico y tratamiento del asma se basaba en la historia clínica y la exploración física. Más recientemente se ha reconocido que las mediciones objetivas de la obstrucción de las vías respiratorias por medio de pruebas de función pulmonar son esenciales en el manejo del asma $(7,8)$.

La prueba más utilizada es la curva Flujo-volumen (9). El Flujo Espiratorio Máximo (PEF), se obtiene en la fase espiratoria de dicha curva, y registra cuán rápidamente se puede eliminar el aire desde la capacidad vital; el PEF registra el momento del máximo flujo aéreo en $\mathrm{Lt} / \mathrm{seg}$ o Lt/min y nos puede orientar si la vía aérea está libre u obstruída (Figura $\left.\mathrm{N}^{\mathrm{o}} 1\right)(4,10,8)$; apenas empiece el estrechamiento causado por el asma, habrá mayor dificultad en la eliminación del aire desde la capacidad vital y, por consiguiente, una disminución en la velocidad del flujo aéreo $(9,11,12)$. El PEF puede ser medido con aparatos portátiles, lo que abarata los costos del estudio y posibilita el autocontrol por los pacientes (13-15). 


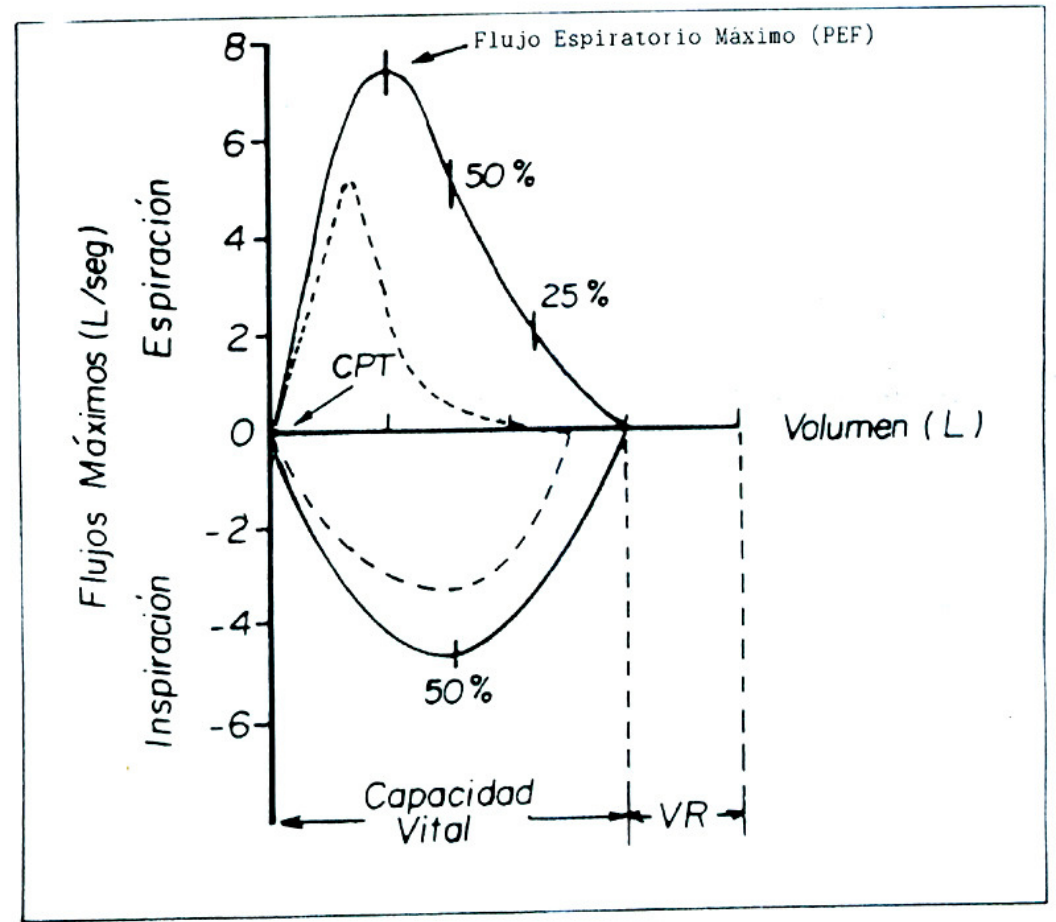

Figural-Curva Flujo- Volumen

Se plantea como hipótesis que las variaciones diurnas del Flujo Espiratorio Máximo (PEF), constituyen una característica del asma bronquial, siendo los objetivos del trabajo: determinar las variaciones diurnas del PEF en el niño asmático en período intercrítico y en segundo lugar buscar la relación existente entre severidad del asma y el grado de variación diurna del PEF.

\section{MATERIALES Y METODOS}

El presente trabajo se realizó en el Hospital Regional Honorio Delgado de la Ciudad de Arequipa, durante los meses de julio de 1992 a junio de 1993. El estudio es prospectivo, analítico, comparativo, e incluye a 38 niños asmáticos (CASOS) y en forma correspondiente a 38 niños sanos (CONTROLES), de ambos sexos y comprendidos entre los 5 y 15 años de edad.

Se contó con la aprobación del Departamento de Pediatría del Hospital y con la aceptación escrita de los padres y/o tutores de los niños. La muestra de niños asmáticos se obtuvo del registro de atenciones del Servicio de Emergencia del Hospital Honorio Delgado. Durante 1 año, se consignó 68 atenciones por crisis asmática, sobre un total de 7,107 atenciones ( $0.96 \%$ del total). Se atendieron 51 niños, siendo 28 de sexo masculino y 23 de sexo femenino. No ingresaron al estudio 13 niños: 4 por ser menores de 5 años, en el caso de 1 niño no se obtuvo el permiso de los padres para realizar el estudio y en el otro caso ocurrió negativa luego de iniciado el mismo; 1 niño no cumplía los requisitos para ser catalogado 
como asma bronquial y en 6 casos no se ubicó el domicilio registrado. Finalmente ingresaron al estudio 38 niños asmáticos.

Los niños sanos se tomaron al azar de pacientes de consultorio externo u hospitalizados en el Servicio de Pediatría y/o Cirugía Pediátrica, con la condición de ser eurtóficos y no sufrir de patología respiratoria en el momento del examen, o antecedentes de enfermedad respiratoria crónica y/o alergias respiratorias. Dicho grupo fue similar en edad y sexo al grupo de niños asmáticos.

El estudio se llevó a cabo en el período intercrítico de la enfermedad, realizando visita domiciliaria a cada uno de los niños para obtener los registros del PEF. Se elaboró un cuestionario en base a criterios clínicos aceptados, para catalogar a cada niño asmático en determinado grado de severidad de asma bronquial $(12,16,17)$. Se consideró como asma bronquial, si el niño presentó 3 ó más episodios de sibilancias, con o sin dificultad respiratoria, que no pudieron ser atribuídos a ninguna otra etiología $(12,18)$. En cada caso se dejó constancia de tratamientos recibidos con anterioridad para el asma, especificando la forma de administración de los mismos.

Cada niño fue hallado, para poder realizar las comparaciones con su PEF teórico; las determinaciones del PEF se hicieron con el mini-Wright Peak Flow Meter (Clement Clarke International Ltd). Se instruyó a cada niño sobre el procedimiento a realizar, registrando el valor más alto obtenido en tres intentos $(16,19)$.

Para analizar las variaciones del PEF se elaboró una hoja gráfica, en la que se consignó los valores del mismo, en dos registros diarios a las 6 y 18 horas, durante 7 días consecutivos. Los índices de variabilidad del PEF se obtuvieron de la siguiente manera:

$$
\text { Variabilidad }=\text { valor mayor }- \text { valor menor } \times 100 / \text { valor mayor }
$$

Ejemplo: PEF a las 18 horas $=400=350 \mathrm{PEF}$ a las 06 horas $=350$

$$
\text { Variabilidad }=400-350 \times 100=12.5 \% / 400
$$

Durante el tiempo de estudio, y dos días antes, el niño en forma ideal no recibió tratamiento farmacológico alguno, y en caso de necesitarlo, se reportó en la hoja gráfica respectiva, así como los síntomas que presentaron los pacientes. En todos los casos el niño no realizó esfuerzo físico intenso una hora antes de la flujometría.

\section{Análisis Estadístico}

Para el análisis de los resultados se registró las variaciones porcentuales diarias del PEF (Variabilidad), obteniendo luego de los 7 días de medición, un promedio final de las mismas, que es el que se consigna para cada niño. Para el análisis se utilizaron porcentajes (\%), desviación standard (SD) y $\mathrm{t}$ de Student. La figura $\mathrm{N}^{\circ} 2$, muestra los valores de referencia del PEF (20). 


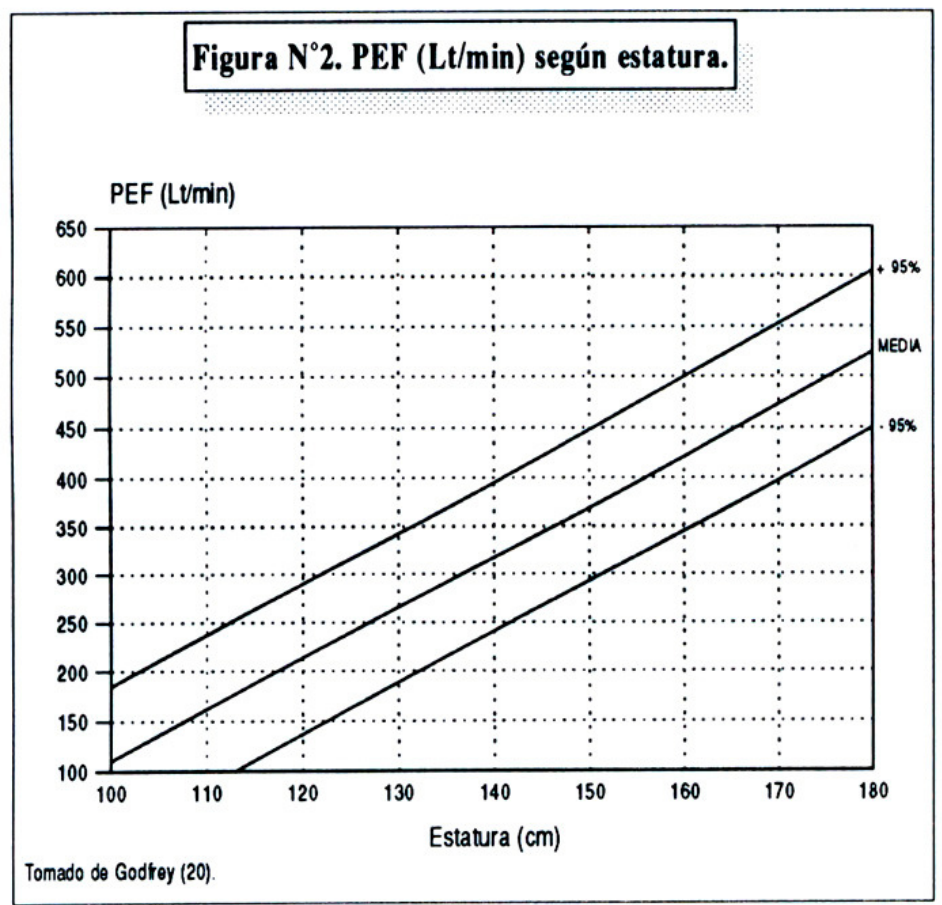

\section{RESULTADOS}

La tabla $\mathrm{N}^{\circ} 1$, muestra la variabilidad del PEF en niños normales, luego de completados los 7 días de registro. Se aprecia que la variabilidad no fue mayor del 9.5\% siendo el promedio global de $4.31 \% \pm 2.19$.

\begin{tabular}{|c|c|c|c|c|c|}
\hline $\mathrm{N}^{*}$ & Variabilidad & $\mathrm{N}^{*}$ & Variabilidad & $\mathrm{N}^{*}$ & Variabilidad \\
\hline 1 & 8.49 & 16 & 2.96 & 31 & 3.81 \\
\hline 2 & 8.00 & 17 & 5.82 & 32 & 1.94 \\
\hline 3 & 9.29 & 18 & 1.77 & 33 & 7.80 \\
\hline 4 & 6.16 & 19 & 4.74 & 34 & 4.24 \\
\hline 5 & 4.05 & 20 & 3.10 & 35 & 2.81 \\
\hline 6 & 3.53 & 21 & 3.74 & 36 & 4.22 \\
\hline 7 & 6.87 & 22 & 3.23 & 37 & 2.27 \\
\hline 8 & 1.41 & 23 & 2.83 & 38 & 2.20 \\
\hline 9 & 3.17 & 24 & 4.00 & & \\
\hline 10 & 7.22 & 25 & 3.31 & & \\
\hline 11 & 8.43 & 26 & 3.35 & & \\
\hline 12 & 1.66 & 27 & 1.38 & & \\
\hline 13 & 4.44 & 28 & 6.96 & & \\
\hline 14 & 4.57 & 29 & 3.42 & & \\
\hline 15 & 5.20 & 30 & 2.37 & & \\
\hline
\end{tabular}


La tabla $\mathrm{N}^{\circ} 2$, muestra la variabilidad del PEF en niños con asma leve, moderado y severo. Se aprecia la tendencia al incremento progresivo en la variabilidad según la severidad de la enfermedad, siendo los valores de $11.37 \% \pm 3.96,20.41 \% \pm 7.67$ y $25.33 \pm 6.11$, respectivamente.

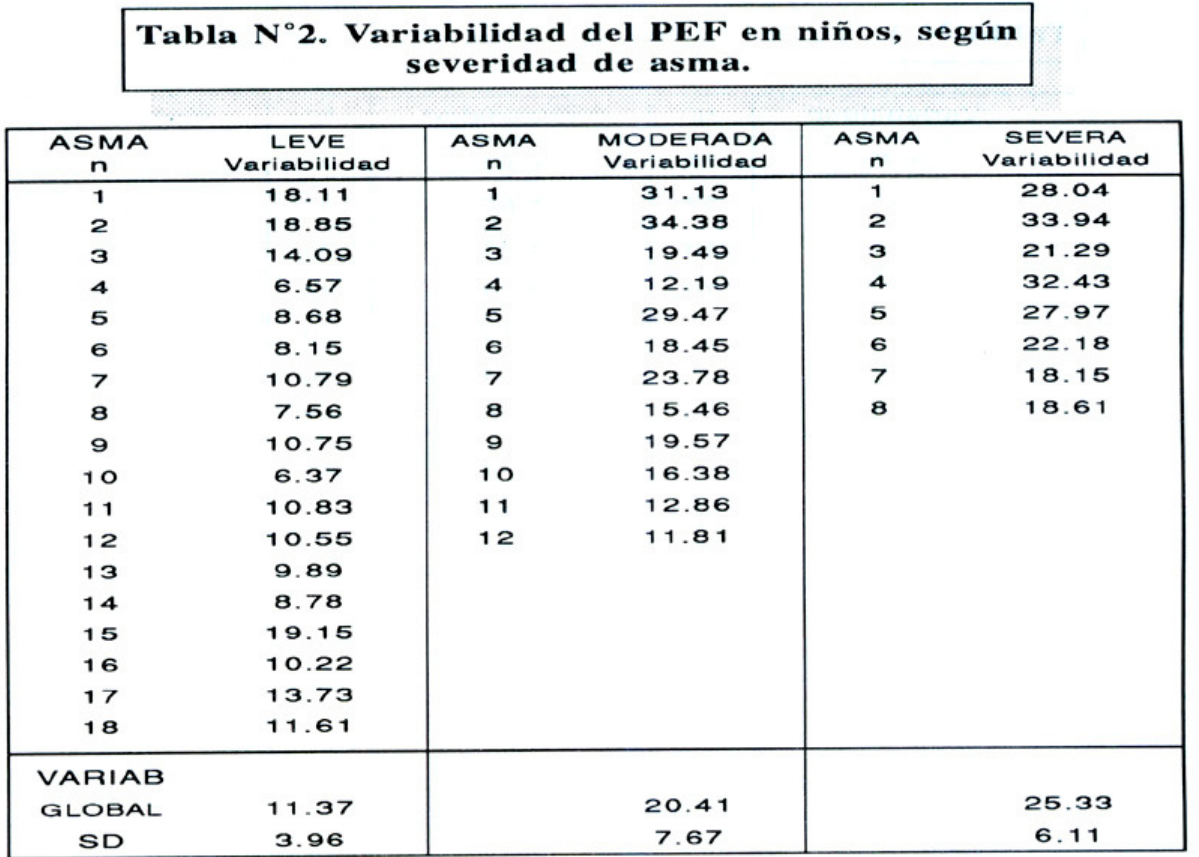

La figura $\mathrm{N}^{\circ}$, muestra de manera gráfica la diferencia en la variabilidad del PEF en los 4 grupos de estudio.

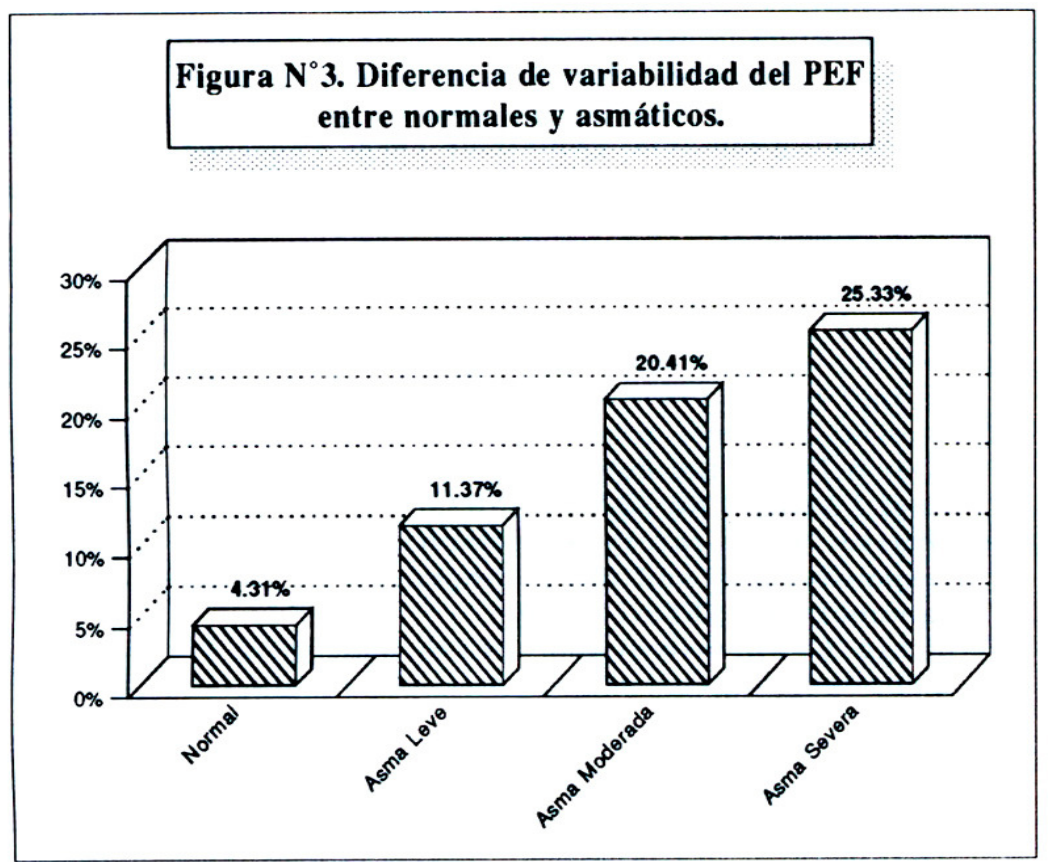


La figura $\mathrm{N}^{\circ} 4$, muestra el porcentaje de niños asmáticos que caen por debajo del Límite Inferior de Normatividad (LIN) (8), de acuerdo al \% del PEF teórico. La comparación se hizo con el promedio de las 06 horas.

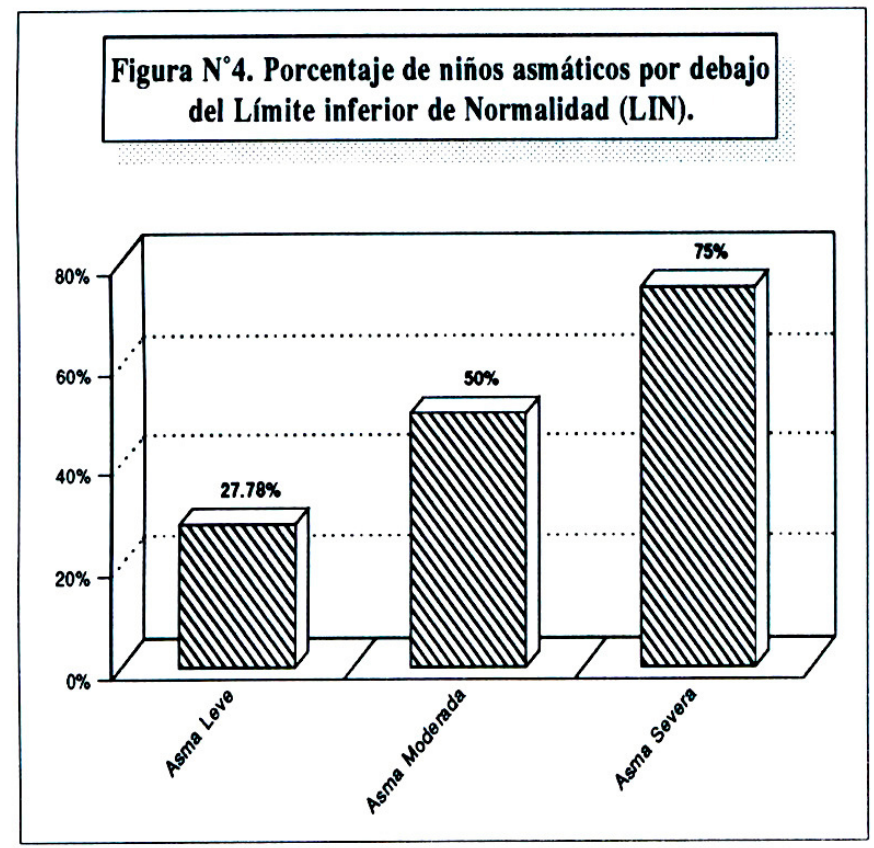

Finalmente, la tabla $\mathrm{N}^{\mathrm{o}} 3$, compara estadísticamente el promedio de variabilidad del PEF obtenido entre los 4 grupos de estudio: niños normales, asma moderado y asma severo.

\begin{tabular}{|c|c|c|c|}
\hline \multicolumn{4}{|c|}{$\begin{array}{l}\text { Tabla N } 3 \text {. Comparación de la variabilidad global } \\
\text { entre los } 4 \text { grupos de estudio. }\end{array}$} \\
\hline & $\begin{array}{l}\text { ASMA } \\
\text { LEVE }\end{array}$ & $\begin{array}{c}\text { ASMA } \\
\text { MODERADO }\end{array}$ & $\begin{array}{c}\text { ASMA } \\
\text { SEVERO }\end{array}$ \\
\hline NORMAL & $\begin{array}{c}\mathrm{t}=8.5945 \\
\mathrm{p}<0.000001 \\
\text { AS }\end{array}$ & $\begin{array}{c}\mathrm{t}=11.7193 \\
\mathrm{p}<0.000001 \\
\text { AS }\end{array}$ & $\begin{array}{c}\mathrm{t}=17.0796 \\
\mathrm{P}<0.000001 \\
\text { AS }\end{array}$ \\
\hline LEVE & 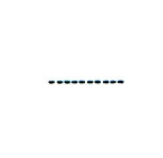 & $\begin{array}{c}t=4.2440 \\
P<0.00021 \\
\text { AS }\end{array}$ & $\begin{array}{c}t=6.9966 \\
P<0.000001 \\
\text { AS }\end{array}$ \\
\hline MODERADO & $\cdots-\cdots$ & $\cdots+\cdots$ & $\begin{array}{c}t=1.5135 \\
p=0.1475 \\
\text { NS }\end{array}$ \\
\hline
\end{tabular}




\section{DISCUSION}

Todos los niños asmáticos y la gran mayoría de niños normales mostraron un patrón de variación del PEF, con los valores más bajos por la mañana (06 horas). Existen muchas teorías para explicar este patrón (12); el valor mínimo del PEF ocurre alrededor de las 4h cuando los niveles de histamina en plasma son elevados y los niveles de AMPc y epinefrina están bajos; los niveles de cortisol plasmático llegan a su mínimo alrededor de medianoche. Otros postulan un aumento del tono vagal y una disminución del aclaramiento mucociliar por la noche, relacionándolos con el descenso nocturno del PEF.

La amplitud promedio de variación del PEF en niños normales fue $4.31 \%$; este valor es bajo comparado con otros reportes que refieren amplitud de variación de alrededor de $8.2 \%$ $(5,7,8,14,19)$.

Existen varios factores que explican esta diferencia: la forma de determinar la variabilidad es importante, ya que en el denominador (ver fórmula) se puede considerar el valor máximo pero también el valor máximo pero también el valor promedio del PEF, por lo tanto muchas veces los resultados no son comparables. Por otro lado, la muestra de niños normales es seleccionada, puesto que se descartó en estos niños antecedentes de enfermedad respiratoria importante y/o alergias respiratorias, siendo otra condición el de no sufrir de patología respiratoria en el momento del estudio.

La influencia del medio ambiente es importante (3), habiéndose demostrado que la residencia en áreas con niveles altos de polución atmosférica o la convivencia en el hogar de padres fumadores tiene una influencia significativa en los niveles del PEF (3,21-24). Tal vez importe también la disminución de la exposición al ácaro de polvo habitación que se desarrolla con facilidad en lugares húmedos y tibios, y se multiplica mejor cuando existe una humedad relativa del aire de $75-80 \%$ y una temperatura de por lo menos $21^{\circ} \mathrm{C}$, condiciones que no existen totalmente en nuestra ciudad $(12,25)$.

Todos los niños asmáticos mostraron una amplitud de variación del PEF significativamente mayor que la de los niños normales, con una variabilidad global de $11.37 \%, 20.41 \%$ y $25.33 \%$ para el asma leve, moderado y severo respectivamente. En todos los casos se alcanza alta significancia estadística al comparar estos valores con el normal.

Se ha tratado de utilizar la variabilidad del PEF para catalogar de acuerdo a la misma a cada niño asmático en estudio, en determinado grado de severidad de asma bronquial $(10,12,17)$. Al aplicar la desviación estándar (SD) a nuestro resultados, encontramos que los niños asmáticos que previamente habían sido catalogados por parámetros clínicos muestran en forma correspondiente un aumento de la variabilidad del PEF de acuerdo a la severidad de la enfermedad, con rangos que van del $7.41 \%$ al $15.33 \%$ para el asma leve, del 12.74 al $28.10 \%$ para el asma moderado y del $19.22 \%$ al $31.45 \%$ para el asma severo. Como se aprecia, existe diferencia estadística significativa entre los asmáticos leves y los asmáticos moderados y/o severos, pero hay una superposición parcial de los resultados entre asmáticos moderados y severos, si bien la tendencia al incremento en la variabilidad persiste. Al respecto puede haber influído la pequeña muestra de niños con asma severo y al hecho de que 3 de 8 niños (37.50\%) recibían tratamiento permanente para el asma, que no 
se suspendió durante el estudio. La investigación se realizó en el período intercrítico de la enfermedad, encontrando que la inmensa mayoría de niños recibía tratamiento farmacológico sólo al presentar exacerbación de los síntomas o crisis asmática; sólo 4 de los 38 niños recibían medicación en forma sostenida.

Los niños asmáticos que presentaron síntomas durante el período de estudio mostraron correspondiente un incremento en la amplitud de variación del PEF, correlacionado bien con las molestias referidas por el pacientes $(15,26-28)$, e incluso pueden constituir un aviso precoz de un ataque agudo de asma $(12,13,29,30)$.

Tanto en niños normales como en asmáticos se encuentra una tendencia a niveles mayores de variabilidad conforme la edad del niño es menor, otros estudios sugieren que a hiperreactividad bronquial (HRB) es grande en la niñez temprana y disminuye después, explicando este hallazgo $(14,31)$. La severidad del asma bronquial guarda una estrecha correlación con niveles más graves de $\mathrm{HRB}$, por lo que las determinaciones seriadas del PEF son útiles en la valoración y tratamiento del niño asmático $(7,13,16,32)$. En pacientes nuevos puede ayudar a establecer el diagnóstico de asma si no hay broncoconstricción en el momento de la consulta y en asmáticos conocidos sirve para monitorizar el tratamiento y predecir un empeoramiento de la enfermedad (15)(30)(32).

El Límite Inferior de Normalidad (LIN) se define como el valor absoluto sobre el cual se distribuye el 95\% de los sujetos normales (9). Aplicando este concepto hemos encontrado que una determinación aislada del PEF (sólo el promedio de las 06 horas) no es útil en la valoración de la severidad de la enfermedad, ya que de esta manera existiría un alto porcentaje de falsos negativos. Es así, que sólo identificamos a un $27.78 \%$ de asmáticos leves $(5 / 18)$, un $50 \%$ de asmáticos moderados $(6 / 12)$ y un $75 \%$ de asmáticos severos $(6 / 8)$, remarcando la importancia de las determinaciones seriadas del PEF, con por lo menos 2 registros diarios.

Se ha sugerido que una variación diurna del PEF $>$ del 20\% identifica a los sujetos asmáticos en estudio poblacionales; este valor está siendo cuestionado, ya que se ha demostrado que existe en la población una distribución continua de la variabilidad del PEF, con una considerable superposición entre sujetos asmáticos y no-asmáticos, de tal manera que el límite que divide asma y no-asma es desconocido $(7,14)$.

En nuestro medio la mayor variabilidad encontrada en un niño normal fue $9.29 \%(\mathrm{~N}-03)$ y estadísticamente (SD) los asmáticos leves tienen una variabilidad mayor de $7.41 \%$, por lo que postulamos que si bien el rango que separa a un niño normal de otro asmático no es preciso, en un niño con una historia clínica sugestiva, una variabilidad global mayor del $8 \%$ indica que el diagnóstico de asma es altamente probable.

\section{Correspondencia}

Dr. Arturo Recabarren

Av. Militar 1950. Lima 14

Telefono:511- 472-4970. 


\section{REFERENCIAS BIBLIOGRAFICAS}

1.Barnes. New aspects of asthma. J Int Med 1992; 231: 403-461.

2.Cruz Mena. Aparato respiratorio, fisiología y clínica. 3ra. Edición. Santiago:Editorial Mediterráneo. 1990.

3.Girardi. Asma bronquial en el niño. Rev Chil Pediatría 1982. 53: 371-379.

4.Gregg. Peak Espiratory Flow in normal subjects. Br Med J 1989; 298: 1068-1070.

5.Higgins, Britton, Chin. The distribution of Expiratory Flow variability in a population sample. Am Rev Respir Dis 1989; 140: 1368-1372.

6.Knudson, Lebowitz, Holberg. Changes in the normal maximal expiratory Flow-volume curve with growth and aging. Am Rev Respir Dis 1983; 127: 725-734.

7.Hetzel. Variaciones diurnas en el Flujo Espiratorio Máximo, ¿una característica diagnóstica?. Triángulo; 27(3): 17-26.

8.Moreno y Oyarzún. Recomendaciones sobre informe espirométrico. Enferm Respir Tórax 1988; 4: 138-149.

9.Oyarzún. Estudio funcional respiratorio I. Test Ventilatorios. Rev Med Chile 1983; 111: 862-868.

10.Lenfant. Objetive measures of lung function. All Clin Inmunol 1991; 88(3): 439-446.

11.Ancic. Enfermedades respiratorias, rol del laboratorio. 2da. Edición. IMC Ediciones Científicas, Santiago 1990.

12.Tinkelman. Chilhood Asthma. 2da. edición. Mercer Dekker INC, New York 1993.

13.Lebowitz. The use of Peak Expiratory Flow Rate measurements in respiratory disease. Pediatric Pulmonol 1991; 166-174.

14.Quachenboss, Lebowitz. The normal range of diurnal changes in Peak Expiratory Flow Rates. Am Rev Respir Dis 1991; 143: 323-330.

15.Sly, Landau. Home recording of Peak Expiratory Flow Rates and perception of asthma. Am J Dis Child 1985; 139: 479-482.

16.Lenfant. Definition and diagnosis of astma. All Clin Inmunol 1991; 88: 427-435.

17.Girardi. Programa de obstrucción bronquial e IRA en el niño. Comité Técnico de Enfermedades Respiratorias Infantiles. Santiago. 1991.

18.Freyre Rebaza. Escala de riesgo para el desarrollo ulterior de episodios de sibilancias y de asma bronquial en niños. Acta Médica Peruana 1992; 16: 93-99.

19.Lebowitz, Knudson \& Robertson. Significance of intraindividual changes in Maximun Expiratoriy Flow Volume and Peak Expiratory Flow measurements. Chest 1982; 81: 566571.

20.Godfrey. Peak Expiratory Flow Rate. Brit J Dis Chest 1970; 64: 15-24.

21.Bello. Efectos de la contaminación atmósferica sobre el sistema respiratorio. Enf Tórax 1988; 4: 151-158.

22.Martinez, Cline. Increased incidence of asthma in childreen of smoking mothers. Pediatrics 1992; 89: 21-26.

23.Perrin, Lagier. Occupational asthma, validity of monitoring of Peak Expiratory Flow Rates and non-allergy bronchial responsiveness as compared to specific inhalation challenge. Eur Respir J 1992; 5: 40-48.

24.Young. The influence of a family history of asthma and parent smoking on airway responsiveness in early infancy. N Engl J Med 1991; 324: 1168-73. 
25.Sporik, Holgate. Exposure to House-Dust Mite Allergen (Dep pI) and the development of asthma in childhood. N Engl J Med 1990; 323: 502-507.

26.Busse. Respiratory infections, their role in airway responsiveness and the pathogenesis of asthma. All Clin Inmunol 1990; 85: 671-683.

27.Gregg. Can measurement of Peak Expiratory Flow enhance compliance in chronic asthma? Eur Respir J 1992; 5: 136-138

28.Hogg. Persistant and latent viral infections in the pathology of asthma. Am Rev Respir Dis 1992; 145: 57-59.

29.Larsen. Asthma in children. N Engl J Med 1992; 326: 1540-1545.

30.Bellia, Cibella. Characteristics and prognostic value of morning dipping of Peak Expiratory Flow Rate in stable asthmatic subjects. Chest 1988; 1: 89-94.

31.McFadden. Pathogenesis of asthma. All Clin Inmunol 1986; 73: 413-424.

32.Tsanakas. Peak Expiratory Flow Rate in treating patients. Am J Dis Child 1985; 139: 738-742. 